\title{
Improved SIFT algorithm for place categorization
}

\begin{abstract}
The main aim of this paper is an improvement of the famous Scale Invariant Feature Transform (SIFT) algorithm used in place categorization. Masking approach to reduce the computational complexity of SIFT have been proposed. Tradeoff between key points and processing time on feature extraction has been used. Selected parameters used in the experiment demonstrated that the computational cost of SIFT in feature extraction can be reduced to half. The categorization performance techniques of the masked image achieved good accuracy of more than $80 \%$ and further experimental results on classification using nearest neighbor achieved good results. The proposed method will help to minimize computation cost in SIFT thereby, improving the performance of robotic mapping, navigation, and matching.
\end{abstract}

Keyword: SIFT algorithm; Place categorization 